\title{
Mirando al Estado desde la intersectorialidad y la intergubernamentalidad: El caso de la Iniciativa Aprende Saludable ${ }^{+\$}$
}

\author{
RICARDO CUENCA* \\ Instituto de Estudios Peruanos \\ rcuenca@iep.org.pe \\ SANDRA CARRILLO** \\ Instituto de Estudios Peruanos \\ scarrillo@iep.org.pe \\ FÉLIX PUÉMAPE*** \\ Instituto de Estudios Peruanos \\ fpuemape@iep.org.pe \\ https://doi.org/10.18800/rcpg.201602.009
}

\section{Resumen}

En los últimos años, en el Perú se han ido forjando experiencias que han intentado buscar integración sectorial y coordinación intergubernamental. Este es el caso de la Iniciativa «Aprende Saludable», que desde 2013 ha buscado articular programas sectoriales de los Ministerios de Salud y de Desarrollo e Inclusión Social a las estrategias del Ministerio de Educación para la mejora de los aprendizajes de los estudiantes. El objetivo del artículo es analizar las posibilidades y los límites de la puesta en marcha de políticas públicas intersectoriales e intergubernamentales. Para ello se estudió la implementación de Aprende Saludable desde un enfoque cualitativo con el método de estudio de caso. El resultado principal es que Aprende Saludable es más una iniciativa de coordinación intersectorial, antes que una experiencia de intersectorialidad, apoyada en esfuerzos individuales más que institucionales. En ese sentido, ilustra las barreras de diseño institucional del Estado en un contexto de descentralización y los enfrentamientos de distintas tradiciones y culturas institucionales sectoriales.

Palabras clave: Estado, intergubernamentalidad, intersectorialidad, descentralización.

\footnotetext{
* Doctor en Educación por la Universidad Autónoma de Madrid. Director del Instituto de Estudios Peruanos.

** Magíster en Políticas Públicas y Sociales por la Universidad Pompeu Fabra (UPF).

*** Licenciado en Ciencia Política y Gobierno por la Pontificia Universidad Católica del Perú (PUCP).

+ Recibido el 27 de octubre de 2016; aceptado el 5 de febrero de 2017.

\$ Este artículo se realizó en el marco de la consultoría «Evaluación y propuesta de fortalecimiento del proceso de institucionalización de Aprende Saludable a nivel intersectorial nacional, regional y local» auspiciada por el Banco Interamericano de Desarrollo en el año 2015.
} 
Looking at the State from intersectorality and intergovernmentalism: The Case of the Healthy Learning Initiative

\begin{abstract}
In recent years, there have been forged in Peru years' experience who have tried to move towards sectoral integration efforts and intergovernmental coordination. This is the case of Aprende Saludable an initiative that since 2013 has sought to articulate sectoral programs from Ministry of Health and Ministry of Development and Social Inclusion with the strategies of the Ministry of Education to improve student learning. The aim of the paper is to analyse the possibilities and limits of the implementation of public policies oriented to inter-governmental and inter-sectorial approaches. This requires the implementation of «Aprende Saludable» was studied from a qualitative approach with case study method. The main result is that «Aprende Saludable» is an initiative of inter-sectorial coordination, rather than an inter-sectorial experience, based on individual rather than institutional efforts. Barriers represent institutional design of the government in a context of decentralization and clashes of different traditions and cultures institutional sector.
\end{abstract}

Key words: Government, decentralization, inter-governmentality, inter-sectorial. 


\section{INTRODUCCIÓN}

En el Perú, pese a las tendencias hacia la sectorialización y la centralización que han existido históricamente, se han ido forjando en los últimos años experiencias que han intentado transitar hacia esfuerzos de integración sectorial y coordinación intergubernamental. Algunas de ellas, provenientes del sector educación, resultan interesantes de analizar, pues este sector fue uno de los que vivió con más intensidad el proceso de descentralización (Cuenca, 2013). Aunque el balance general parece haber sido positivo, falta que se definan asuntos institucionales y políticos asociados al proceso (Chirinos, 2012; Muñoz y Valdivia, 2012; Molina, 2010).

Bajo esas premisas, una serie de trabajos han investigado las experiencias de coordinación intersectorial e intergubernamental en el Perú. Por ejemplo, Carrillo y Reátegui (2012) trabajaron el caso de la implementación del proyecto de inversión pública «Mejoramiento de los niveles de logro de aprendizajes en comunicación, matemática y personal social en los niños y niñas del II y III ciclo de educación básica regular pública» del distrito de Julcán, en el departamento de La Libertad, el cual estaba basado en un esfuerzo de coordinación intergubernamental entre el Gobierno regional y el Municipio Distrital. Las autoras encontraron que pese a que el Gobierno regional veía como interlocutores válidos a los gobiernos locales y había emprendido una reforma institucional orientada a mejorar mecanismos de coordinación con otras instancias, este no generó espacios realmente funcionales para la coordinación intergubernamental. Una razón de ello es que sus funcionarios consideraban a los municipios como instancias altamente politizadas cuyos actores respondían a ambiciones políticas inmediatas antes que a planes de largo aliento que beneficien a toda la comunidad.

Por otro lado, Pezo (2012) estudió la experiencia del «Plan Mancomunado para el desarrollado educativo y social del distrito de Cuńumbuqui» en San Martín, llevado a cabo durante los años 2011 y 2012. Encontró que pese a que los planes habían logrado importantes avances en torno a un modelo de gobernanza territorial — los cuales se habían conseguido en buena parte por el fuerte tejido social existente en la zona, lo que significó un contexto favorable para la coordinación intergubernamental—, también evidenciaban una serie de limitaciones.

En paralelo, Muñoz, Neyra y Vargas (2012) investigaron la gestión educativa municipal en el distrito de La Matanza, en Piura. Este caso constituyó un ejemplo de gestión concertada a nivel local con otros niveles de gobierno, dado 
que se desmarcó de los esquemas de gobiernos locales, generalmente orientados por el obrismo antes que por políticas de largo plazo, y elaboró diversas iniciativas en gestión educativa descentralizada. Así, se evidenció que a pesar del énfasis del alcalde en este distrito para implementar eficientes instancias de coordinación, tanto con la Unidad de Gestión Educativa Local (UGEL) como con la sociedad civil, la coordinación con el nivel nacional fue inexistente y con el nivel regional muy incipiente.

Una de las más recientes experiencias desde el Estado por impulsar políticas públicas orientadas hacia una gestión intersectorial e intergubernamental ha sido la Iniciativa «Aprende Saludable». Esta se gestó a inicios de 2013, cuando el Ministerio de Educación (Minedu) — desde el Programa de Mejora de los Aprendizajes—, el Ministerio de Desarrollo e Inclusión Social (Midis) —desde el Programa Nacional de Alimentación Escolar Qali Warma- y el Ministerio de Salud (Minsa) — desde el Plan de Salud Escolar- acordaron desarrollar una iniciativa intersectorial e intergubernamental para beneficiar y potenciar el logro de aprendizajes de los escolares peruanos del nivel primario (Minedu, s/f). Así, a partir de 2013 se publicaron diversas normas con la finalidad de articular las actividades de los sectores en torno a la Iniciativa, la cual fue ratificada en diciembre de 2013 por los ministros de los tres sectores en el marco de la Estrategia Nacional «Incluir para crecer» ${ }^{1}$.

Aprende Saludable, como todas las iniciativas de coordinación, es importante en la medida que reconoce que el logro integral de las políticas públicas depende cada vez menos de un solo sector. Si bien en los últimos ańos el Ministerio de Educación ha ido superando una serie de deficiencias — lo cual se ha visto reflejado en la mejora de la puntuación del Perú en la más reciente prueba Pisa- para la optimización de los servicios que brinda, ha sido necesario que trabaje de la mano con otros sectores y niveles de gobierno. Lo mismo sucede cuando se plantea la solución de otros problemas comunes, como por ejemplo la seguridad, la cual no solo depende de que el Ministerio del Interior depure la Policía de malos elementos, invierta en mejorar la inteligencia policial o compre más patrulleros, sino también de que otros sectores puedan trabajar en una política preventiva que reduzca la incidencia criminal en determinados sectores vulnerables. Así, el costo de no trabajar en conjunto es el estancamiento de los resultados conseguidos por cada sector por sí solo. Ante ello, es imprescindible entender los factores que facilitan y limitan tales sinergias.

1 Ver decreto supremo 039-2014-PCM. 
A la luz de la importancia de la coordinación para el desarrollo de políticas públicas integrales, el objetivo de este artículo es analizar las posibilidades y los límites de la puesta en marcha de políticas públicas orientadas hacia enfoques intersectoriales e intergubernamentales. Por su cercanía en el tiempo y el alcance que ha tenido, se estudiará la implementación de la Iniciativa Aprende Saludable, que servirá de caso de investigación.

Partimos de la hipótesis que, pese a la voluntad de una serie de actores para impulsar esta Iniciativa, tanto el contexto institucional en el que se desenvuelven (marcos legales sectoriales, centralistas y disfuncionales) como la cantidad de recursos con los que cuentan (burócratas con escasos recursos políticos, económicos, administrativos e ideológicos) se constituyen en las principales barreras para el desarrollo de iniciativas intergubernamentales y, sobre todo, intersectoriales.

\section{REVISIÓN DE LA LITERATURA}

Para que una política pública² ${ }^{2}$ ueda llevarse a cabo de forma satisfactoria, buena parte de la literatura sobre el tema coincide en que tiene que pasar, por lo menos, por cuatro momentos: agendación, diseño, implementación y evaluación (Dye, 2008; Lindblom, 1991). Sin embargo, recién se ha venido considerando la importancia crucial que tiene la existencia de mecanismos de coordinación para lograr mejores resultados.

La cuestión de la coordinación entre los sectores de un mismo nivel de gobierno — salud, educación, vivienda y otros — se empezó a abordar ante la existencia de un Estado con organizaciones disímiles (Migdal, 1988). Otros desencadenantes para la reflexión en torno a este tema fueron la existencia de problemas que traspasan la esfera de la competencia de una sola oficina, los proyectos que involucran a las ciudades como un todo, la descentralización de la ejecución de políticas sociales y la centralización de la formulación o coordinación de las políticas sociales (Cunill, 2014)3. Los procesos de descentralización regional y local que tuvieron lugar en diversas regiones del mundo, aunque

\footnotetext{
2 En este artículo, tomando la definición de Cortázar (2001), consideramos como política pública al flujo de interacciones y decisiones que implican el empleo de la autoridad pública con la finalidad de responder a situaciones consideradas problemas públicos, es decir, situaciones problemáticas que exigen la acción del Estado (Cortázar, 2001, p. 6).

3 Según esta misma autora, la preocupación por la coordinación se refleja en cuanto, a inicios de la década de 1990, organismos multilaterales empezaron a hablar de políticas sociales integradas en los sectores salud y educación (Cunill, 2014, p. 10).
} 
especialmente en América Latina a finales de la década de 1980, han contribuido a que el tópico de la coordinación haya ido cobrando más importancia.

Paulatinamente, la coordinación entre los sectores de un mismo nivel de gobierno — conocida como intersectorialidad - y entre los diferentes niveles de gobierno de un país — conocida como intergubernamentalidad - comenzaron a considerarse como condicionantes para el desarrollo satisfactorio de políticas públicas. En las siguientes líneas se repasará algunos de los factores que han sido señalados como incentivos y barreras para la coordinación en el Estado tanto a nivel intersectorial como intergubernamental en América Latina y el Perú.

\section{Incentivos y barreras para la coordinación intersectorial}

Ante los retos que impuso el abordaje sectorializado de las políticas públicas, especialmente las de carácter social, la coordinación intersectorial comenzó a estudiarse mucho antes que la coordinación intergubernamental. De esa forma, ordenando las respuestas que desde la literatura sobre el tema se han planteado, un primer grupo tendió a enfatizar la importancia de las instituciones que rigen las organizaciones.

En su revisión sobre el tema, Cunill (2014) repasa un debate existente sobre el alcance de las reglas que promueven la coordinación intersectorial. Así, menciona que autores como Sulbrandt (2005) demostraron que cuando se establecen más instancias institucionalizadas de comunicación horizontal al interior de un Estado, existen mayores oportunidades para el desarrollo de la intersectorialidad. Sin embargo, también hace referencia a otros autores, como Correa (2001), que han llamado la atención sobre el momento en el que las instancias de coordinación se establecen. Sostiene Cunill (2014, p. 22), que según Correa, cuando estos espacios creados para facilitar la relación entre las diversas organizaciones operan con posterioridad a la planificación del trabajo que cada uno de los sectores ha realizado, su accionar se torna estéril (Cunill, 2014, p. 22). La razón detrás de ello reside en que readaptar las organizaciones a nuevas tareas y fines resulta costoso, más aún si los burócratas se ven frente a otras organizaciones que, por lo menos en un primer momento, podrían representar una amenaza a su autonomía.

Con una mirada distinta, Acuña (2005) señala que otro problema que se puede suscitar cuando las instancias de coordinación ya están constituidas es que los ministerios - y en buena medida también muchas oficinas dentro de un mismo ministerio - ven asuntos que se resuelven a través de marcos normativos completamente diferentes. Asimismo, los funcionarios de cada uno de los sectores ya están acostumbrados a tratar con un grupo determinado de actores 
(Acuña, 2005, p. 8). De ahí que el diálogo con actores distintos con premisas diferentes agudice la dificultad para ponerse de acuerdo.

Otro grupo de autores resalta la preponderancia de los actores al momento de entender los incentivos y obstáculos que existen para la coordinación intersectorial. Bardach (1998) refiere que la coordinación se cristaliza solo cuando existen líderes capaces de desarrollar un discurso en el que persuadan a todos los actores que van a ganar algo o, por lo menos, que no van a perder nada. En esa línea, Bryson, Crosby y Stone (2006) consideran que la coordinación tiene lugar solo cuando los liderazgos toman diversos roles, tales como patrocinadores, articuladores o facilitadores de la coordinación (Bryson, Crosby y Stone, 2006, p. 5).

Complementando la literatura presentada, Repetto (2005) explica que para entender la presencia o ausencia de coordinación entre los sectores es necesario conocer los recursos que cada uno tiene. Según este autor, los recursos definen la capacidad política de los actores para negociar o para ejercer acciones de veto (Repetto, 2005, p. 53). Así, menciona que se deben tener, por lo menos, cuatro tipos de recursos: políticos, económicos, administrativos e ideológicos. Los primeros hacen referencia al apoyo de la ciudadanía, de grupos de interés y de autoridades políticas, así como a la posesión de autoridad formal e informal, liderazgo, capacidad para movilizar personas y para negociar, argumentar y comunicar. Cuando se habla de recursos económicos, se hace referencia a presupuestos y, en general, a la posesión de dinero para incidir en la agenda gubernamental. Poseer recursos administrativos implica tener mandato legal, organización, información, experiencia técnica y capacidad para descifrar el contexto. Finalmente, los recursos ideológicos hacen referencia a ideas, valores, prestigio moral y concepciones del mundo. En el marco de la coordinación, este autor afirma que, con sus recursos, los actores construyen coaliciones políticas.

Cuando los actores tienen recursos, pueden lidiar con las lógicas sectoriales que constantemente están presentes en el Estado, haciendo que la coordinación tenga mayores posibilidades de cristalizarse. Sin embargo, para este autor, una mirada centrada en los actores y su falta de capacidad política y recursos resulta insuficiente para entender los límites de la coordinación intersectorial, por lo que también desarrolla la disonancia que muchas veces existe entre las instituciones formales e informales.

De esa manera, plantea que las reglas formales de baja calidad amplían las probabilidades que determinados actores conserven una lógica sectorial, más aún en contextos en los que tienden a primar reglas informales. Para Repetto (2005), las reglas de baja calidad se manifiestan cuando las reglas formales abundan y 
permanecen totalmente desligadas de las costumbres y las prácticas culturales. De ahí que rescata la importancia de reducir significativamente la brecha entre la regla formal y la regla informal, sin afectar el potencial de flexibilidad e innovación que suelen tener las normas no escritas (Repetto, 2005, p. 46).

Dada la complejidad que reviste el Estado, los incentivos y las barreras para la coordinación dependen de múltiples factores. Estos giran en torno a dos aspectos: institucionales y de agencia política. Así, aunque mecanismos formales que normen la coordinación pueden facilitarla, se pueden ver limitados por el momento en el que son expedidos, sus diferencias con los de otros sectores, su distanciamiento de la realidad o su carácter sectorial. Por otro lado, aunque hay actores que pueden impulsar la coordinación, estos podrían ver frenados sus intentos si es que tienen pocos recursos o si ven a los otros sectores como inferiores.

\section{Incentivos y barreras para la coordinación intergubernamental}

La coordinación intergubernamental es un tópico relativamente reciente en América Latina 4 . Sin embargo, en muchos casos reviste una complejidad mayor a la coordinación intersectorial, dado que implica que los sectores ya no solo tienen que coordinar entre ellos sino también con los funcionarios de los mismos sectores en los otros niveles de gobierno. Los autores que han procurado explicar los problemas que acarrea han enfatizado predominantemente las variables institucionales.

Desde una mirada muy epidérmica, aunque útil para entender la naturaleza de las relaciones entre niveles de gobierno, Nickson (1998) plantea que las relaciones entre niveles de gobierno reflejan el choque entre dos formas de gobierno: centralización y descentralización. El primero implica la sectorialización de las políticas públicas, mientras que el segundo su territorialización, entendiendo este concepto como que toda prestación de servicios debe pensarse desde los niveles subnacionales antes que desde el Estado central. La centralización y la descentralización implican, a su vez, dos modelos políticos completamente distintos; uno que busca la reducción de costos en la prestación de los bienes públicos y otro que busca la canalización de los intereses de los distintos actores locales. De esta manera, cuando priman en el Estado lógicas más centralizadoras, la coordinación intergubernamental tiende a fracasar ${ }^{5}$.

\footnotetext{
4 Aunque en otras regiones sí se ha problematizado con mayor antelación. Ver Agranoff (1997).

5 Jordana (2001) también utiliza un esquema de análisis similar; basándose en el trabajo de Cohen y Peterson (1997), utiliza los conceptos de pluralismo institucional y monopolio institucional del Estado. El primer tipo de relaciones intergubernamentales implica la existencia de varios mecanismos formales de negociación entre niveles para resolver las
} 
Sin embargo, a la luz del caso colombiano, Meade y Gershberg (2010) argumentaron que el factor que determinó una relación fluida entre los niveles nacional y subnacional fue la existencia de reglas claras respecto al papel de cada sector en el desarrollo de las políticas públicas. Así, para que exista una mayor y mejor coordinación de los esfuerzos en materia educativa, desde inicios de la década de 1990 —específicamente en 1993 y 2001, en un contexto de alta insatisfacción social — el Gobierno de ese país reestructuró y modernizó su sistema administrativo, buscando que los fondos que otorgaba fueran utilizados de mejor manera. Así, especialmente en 2001, se definieron claramente las funciones de cada actor.

Para Jordana (2001), los procesos de coordinación intergubernamental revisten una mayor complejidad. Esta complejidad la relaciona con el hecho de que en una democracia las relaciones intergubernamentales se articulan a través de distintas reglas, formales e informales, que se pueden dividir en tres categorías: estructuras institucionales orientadas a la coordinación y el control, que inciden en la toma de decisiones de los cargos públicos; reglas electorales, que afectan la actividad de los partidos políticos en distintos niveles de gobierno; y reglas de funcionamiento de las administraciones públicas, que influyen sobre el comportamiento de los técnicos y profesionales encargados de gestionar las distintas políticas públicas que se implementan en el territorio (2001, p. 15).

Dicho todo lo anterior, aunque la coordinación intergubernamental puede estar incentivada por reglas claras que definan funciones específicas para cada actor y que protejan la autonomía de los estamentos subnacionales, algunos factores puntuales que pueden estar detrás de la ausencia de coordinación intergubernamental son, además de la poca disposición del Estado central para ceder poder, la ausencia de instancias explícitamente formalizadas para la toma de decisiones conjuntas, lo cual hace que las instancias informales, tendientes a la centralización, primen; la ausencia de representación de los intereses subnacionales en los sistemas electorales, lo cual permite que los partidos nacionales monopolicen el debate político en torno a sus intereses patrimonialistas; y la patrimonialización de la administración pública, que impide el desarrollo de funcionarios estatales de carrera que propicien la coordinación.

exigencias de coordinación. En el segundo tipo se intenta mantener al mínimo las necesidades de coordinación formal. Cuando se desarrollan, suelen adoptarse sistemas simples de negociaciones, los que generalmente no están basados en procedimientos claramente especificados. Así, en un país con un modelo de descentralización basado exclusivamente en monopolios institucionales distribuidos, plantea que el tejido de relaciones necesario será mucho más reducido que en otros casos. 


\section{Nota METOdológica}

La investigación se enmarcó en un enfoque cualitativo y el abordaje metodológico fue a través del estudio de caso. Elegir lo particular permite alcanzar mayores niveles de comprensión del problema de estudio (Galeano, 2009). En tal sentido, el estudio de caso se refiere a la recolección, análisis y representación de información sistemática y estructurada sobre un fenómeno particular, a partir de diversas fuentes, entre las que se incluye a los propios sujetos participantes del caso (Simons, 2012). Siguiendo a Stake (1999), el estudio de caso es del tipo instrumental, pues se examina un hecho particular con el fin de proporcionar mayor conocimiento y comprensión sobre el tema, de forma tal que el caso propiamente es secundario.

La información fue levantada a través del análisis de los diversos documentos producidos (informes, normativas, actas, planes de trabajo, presentaciones, etcétera), que sirvió para la comprensión de la Iniciativa, así como para la elaboración de los instrumentos de recogida de datos. La parte fundamental del levantamiento de información se concentró en la realización de entrevistas semiestructuradas a los actores clave, responsables del mismo, de los diferentes niveles, sectores y regiones.

El campo se desarrolló en dos etapas. La primera se hizo en Lima Metropolitana, con autoridades nacionales y responsables de la Comisión Multisectorial (9 entrevistas) y la segunda se llevó a cabo en los 4 departamentos, con las autoridades regionales y sus especialistas (20 entrevistas).

En total se contó con la participación de 29 personas, tanto de Lima (nivel nacional), como de los 4 departamentos seleccionados (nivel regional): Ayacucho, Lima Provincias, Piura y Ucayali, de los 3 sectores involucrados.

Las entrevistas desarrolladas en Lima Metropolitana estuvieron dirigidas a autoridades: las tres exministras que impulsaron esta Iniciativa, la entonces ministra del Midis, los integrantes de la Comisión Multisectorial: Educación, Salud y Desarrollo e Inclusión Social, así como la Secretaría Técnica.

\section{Resultados}

Durante el trabajo de campo todos los actores involucrados en Aprende Saludable se mostraron conscientes de la importancia que tiene esta iniciativa en el buen desarrollo de los estudiantes. A través de las entrevistas se ha podido indagar acerca de los incentivos y, sobre todo, las barreras para la coordinación intersectorial e intergubernamental. 


\section{Intersectorialidad}

En los casos en los que ha tenido lugar, la coordinación intersectorial se ha visto incentivada por actores y normas específicas. En primer lugar, pese a la dificultad que representa toda iniciativa que implique coordinación entre sectores dispares, Aprende Saludable logró salir adelante en un primer momento por medio de la voluntad política de las ministras de Educación, Salud y Desarrollo e Inclusión Social en el año 2013. En las regiones en las que la Iniciativa ha logrado llevarse a cabo con mayor continuidad, pese al inmovilismo que se impuso tras la pérdida de impulso desde Lima, los protagonistas fueron grupos puntuales de especialistas de los tres ministerios. Por ejemplo, en la siguiente cita una funcionaria de Qali Warma describe la labor de una especialista de la Dirección Regional de Educación (DRE) y lo útil que le resulta para el logro de su trabajo:

[...] por decir, cambiaron al director de la DRE, entonces [le decimos a la especialista de la DRE] queremos un espacio, le decimos, para acercarnos y hacerle una presentación de Aprende Saludable. Ya, para cuándo. Para tal fecha, ¿̨no? Y nos ponemos de acuerdo con [la coordinadora regional del Plan de Salud Escolar] que representa al sector salud y vamos y le hacemos llegar la información, ¿no? Un nivel de avance, como venimos trabajando, ¿no? (Qali Warma-Ayacucho).

En estos casos, los especialistas se han abocado a procesos de coordinación informal de corto aliento que, en definitiva, se ha traducido en que vuelvan a emprender las actividades que contemplan su estructura funcional. Como narra una de las funcionarias del nivel nacional:

Lo que yo he podido identificar es que hay una coordinación, de todas maneras, que no ha sido oficializada. Lo que nosotros les hemos pedido es que oficialicen esas reuniones que tienen, ¿̇no?, pero siempre hay una coordinación porque de todas maneras ellos son la parte, por ejemplo de la DRE, son los que tienen que ver y garantizar... convocar a sus reuniones cuando tiene con sus directores para que den estas facilidades, ¿̨no? Y no solamente se reúnen por Salud Escolar, sino porque tienen otras actividades pendientes que están en conjunto, siempre están en coordinación, en ver qué colegios, cómo está llegando el desayuno, los alimentos que les envían... pero lo que había no era oficializado (Minsa).

Con relación a esto, un dato a tener en cuenta es que algunos entrevistados mencionaron que lograron establecer lazos con otros funcionarios de un sector diferente al propio, dado que algunos de sus pares tienen la misma profesión que ellos (equipos multidisciplinarios). Esto es, en algunos Equipos de Educación hay enfermeras, mientras que en algunos Equipos de Salud hay docentes, lo cual permite que se generen lazos que permiten la coordinación. Según comentan dos funcionarios del sector educación: 
Del Plan de Salud netamente, la señora [coordinadora del Plan de Salud Escolar en la región] [...] ella se iba a las Ugeles [Unidades de Gestión Educativa Local] a dar las charlas a los especialistas [...] Lo bueno es que era maestra y también era licenciada en enfermería, entonces, ella sabía articular los procesos (DRE-Ucayali).

En segundo lugar, los actores interesados en la Iniciativa han propiciado una serie de normativas que la ponen en marcha. Así, a través de las entrevistas ha sido posible encontrar que la norma que incentivó que los funcionarios de alto rango y los políticos del nivel nacional decidan establecer un esfuerzo de coordinación fue la Ley General de Educación, la cual exige que las políticas del sector educación deben ser complementadas con el accionar de otros sectores afines. Sobre esto, una entrevistada explicó:

[...] como una de las justificaciones, para el lado del Midis, que era muy importante y súper potente lucir, era que Qali Warma no se hizo porque se le ocurrió al Presidente sino porque está en la Ley General de Educación, es una obligación del Estado frente a los niños, darle los materiales, el servicio y alimentación, ¿no? Entonces había que hacer algo (Midis).

Paralelamente, en algunas regiones, como Lima Provincias y Ayacucho, los funcionarios desarrollaron, a través de sus Gerencias de Desarrollo Social, marcos normativos en los cuales se especificaba claramente las horas de los desayunos y almuerzos, así como la obligación de los directores de las instituciones educativas de recibir al personal de los otros sectores, especialmente del Plan de Salud Escolar, entre otros aspectos. Sobre ello, una especialista cuenta:

[...] con ellos [la DRE] hemos trabajado generando una directiva en la que se estableció el horario del desayuno y también del almuerzo en las instituciones educativas, así como la conformación de los Comités de Alimentación Escolar que debían asumir los directores o los coordinadores o promotores de las instituciones de nivel inicial o PRONOEIS [Programas No Escolarizados de Educación Inicial], que ellos debían asumir el rol de presidentes, del docente el rol de secretario, entonces, como que a través de esa norma sí tenían que dar cumplimiento, ¿¿no? (Qali Warma-Ayacucho).

Si la coordinación al interior de Aprende Saludable ha dependido de la voluntad de actores específicos y del desarrollo de normas que definen claramente las obligaciones de cada uno de los actores involucrados, las barreras para su desarrollo han estado relacionadas con tres factores relacionados con la forma como se estructura el Estado peruano: la sectorialización de los ministerios involucrados en la Iniciativa, las múltiples responsabilidades que tienen que atender los maestros en su práctica cotidiana, y la excesiva rotación de personal que existe a nivel subnacional.

En las primeras entrevistas que se realizaron en la ciudad de Lima, fue posible notar cómo los funcionarios de rango intermedio de los distintos sectores 
involucrados en Aprende Saludable — pese al mandato de la Ley General de Educación y a la orden explícita que recibieron desde la alta dirección de los ministerios para coordinar- no lograron conjugar efectivamente estos esfuerzos de forma sostenida. Esto se debe a las diferencias en el diseño institucional fuertemente sectorializado de cada uno de los ministerios involucrados, lo cual es posible notar a través del uso de diferentes instrumentos para identificar a su población de interés, así como, de forma más general, en las diferentes estructuras organizacionales de cada uno de los sectores, unas más burocratizadas que otras, o más centralizadas o menos territorializadas que otras.

Todas las exministras, así como varios de los funcionarios entrevistados, hicieron referencia a lo problemático que ha sido la construcción de un sistema de información común, clave para que la coordinación sea posible en el largo plazo. En esa línea, según una de las protagonistas:

Una de las cosas más difíciles, sin embargo, ha sido el tema de información. O sea, quedamos en que teníamos que hacer un esfuerzo de información conjunta, las tres [ministras]. En el papel se veía todo bien pero obviamente a la hora de la hora tú sabes que las cosas no funcionan así y Educación tiene un problema de información grave [...] Ahí hay un tema que es para seguir pensando. Igual el SIS [Seguro Integral de Salud] tiene su propio sistema de información, o sea, que se llenen las FUA [Formato Único de Atención], en fin. Pero esa idea que vamos a montarnos la información, ahí yo diría que son una de las cosas que menos funciona y que queda pendiente todavía: sistemas de información (Minsa).

A lo largo del trabajo de campo en las regiones, otro factor que ha desincentivado la coordinación entre los ministerios involucrados en la Iniciativa ha sido la gran cantidad de obligaciones que tienen los maestros y directores de escuela en su labor cotidiana. Este hecho ha llevado a que en algunas de las regiones estudiadas, especialmente en Piura y Lima Provincias, el programa no funcione bien ante la negativa de los maestros de monitorear el accionar, tanto del Plan de Salud Escolar como de Qali Warma, más allá de sus labores en los Comités de Alimentación Escolar, en lo referente a capacitaciones sobre una adecuada nutrición. De esa manera, ante la negativa de los maestros y directores a coordinar, se rompe la cadena que debería unir a los tres sectores desde el lado que los debería estructurar y cada uno empieza a actuar de forma independiente. Sobre ese tema, dos coordinadores del Plan de Salud Escolar de diferentes regiones cuentan sus experiencias con maestros de su región:

$\mathrm{Y}$ eso [el componente participativo de Aprende Saludable] puede promover los profesores, pero los profesores también se quejan, ¿no? «Nosotros no hemos nacido para ser cocineros, para ser médicos, para ser enfermeros. Nuestra función es enseñarle 
al niño» [...] cuando tú te sientas con ellos, te dicen que ellos tienen ya demasiadas responsabilidades y tareas (Diresa-Piura).

Otra resistencia [es] cómo ingresa la brigada. La brigada empieza a evaluar aula por aula, entonces terminan con unas fichitas de regular número, que es el director quien tiene que firmarlas, ahí hay una primera demora, el director tiene otras actividades y ahí está con las fichitas (Diresa-Lima Provincias).

Una funcionaria del Minedu en Lima también mencionó esta variable como determinante para aletargar los esfuerzos de coordinación entre los sectores, especialmente entre los de educación y salud:

Entonces, el personal de educación sigue mirando como que esto (Aprende Saludable) es una tarea de salud y que lejos de ayudar les complica más la existencia. Pero no es eso. Entonces, es un proceso gradual (Minedu).

Un tercer factor, mencionado por todos los entrevistados en las regiones como causante de los entrampamientos al momento de la coordinación entre los tres sectores involucrados en Aprende Saludable, ha sido la alta rotación de personal. Así, si bien no es determinante para la ausencia de coordinación —dado que, por ejemplo, en Ayacucho hay mucha rotación (principalmente de autoridades), pero, pese a ello, la coordinación existe de forma permanente (porque los especialistas de educación y salud se mantienen, así como el equipo de Qali Warma)_, se ha argumentado que esta rotación inhibe que las cosas puedan hacerse de forma más expeditiva. Esto porque hay que estar explicando permanentemente a los nuevos directores regionales o gerentes de desarrollo social sobre Aprende Saludable, lo cual es como empezar desde cero. Más aún, en regiones en las cuales los funcionarios no han asumido en los hechos la importancia de la Iniciativa — dado que a nivel discursivo todos lo hacen-, este factor se hace más crucial para entender la sectorialización del accionar del Minsa, Minedu y Qali Warma. Sobre este tema, una especialista comentó:

[...] hasta diciembre, bien; en enero no había personal, en febrero recién llegaban funcionarios; en marzo, los que pusieron en febrero ya los estaban cambiando; en abril hubo otro cambio; en fin (Qali Warma-Lima Provincias).

La misma inquietud fue recogida en otra entrevista, realizada a especialistas de la región Ucayali, al momento de pedirles recomendaciones para mejorar la Iniciativa:

E: Pero entonces, ahí en esa línea, en la experiencias que ustedes han tenido, en lo que fue y en lo que es ahora, ¿qué recomendaciones se daría para que [Aprende Saludable] funcione a nivel regional?

A1: Primero debería ponerse personas idóneas. 
A2: Yo decía con un perfil.

A1: Con un perfil, debería haber la continuidad del personal, por eso es que continuamos nosotros, porque yo te puedo hablar desde el inicio del plan junto con el maestro, junto con la profesora (DRE-Ucayali).

Corroborar las barreras más inmediatas que han existido para el desarrollo de Aprende Saludable permite entender que la coordinación no es unívoca. A partir de lo trabajado, y en la línea de lo mencionado anteriormente, se han revelado dos dimensiones claramente definidas: una de carácter conceptual y otra de carácter procedimental. Desde la dimensión de carácter conceptual, la coordinación es vista como un proceso determinado por los requerimientos propios de las políticas públicas, principalmente los que están bajo la égida de los ministerios que se encargan de proveer bienes (Salud, Educación y Desarrollo e Inclusión Social).

Por otro lado, desde la dimensión de carácter procedimental, la coordinación es concebida como un proceso normado por una regla específica. Cada una de ellas es imaginada por distintos sectores. La primera, básicamente, por los funcionarios de alto rango de cada uno de estos sectores, muchos de los cuales han empezado a venir, tras la caída del sistema de partidos y las reformas de libre mercado llevadas a cabo durante la década de 1990, de la academia y, más específicamente, de centros de pensamiento especializados en el estudio integral de las políticas públicas. Por su parte, la segunda es sostenida por los funcionarios estatales de rango medio, quienes, ante los incentivos existentes en el Estado y en toda organización a favor de un accionar sectorializado, así como por la naturaleza de los recursos del Estado (en relación con los presupuestos y las jerarquías, por ejemplo), actúan predominantemente sobre la base de procedimientos formales.

En suma, la información recopilada ha permitido notar que factores como la multidimensionalidad subyacente en el planteamiento de la Iniciativa Aprende Saludable y el contexto alrededor de los funcionarios públicos encargados de cristalizarla (entidades históricamente sectorializadas, actores clave con múltiples responsabilidades y alta rotación de cargos) han contribuido a que no pueda institucionalizarse. La ausencia de una convergencia entre la dimensión conceptual de los funcionarios de alto rango y los desincentivos institucionales que determinan el accionar de los funcionarios públicos de rango medio han llevado a que la propuesta se desarrolle de forma lenta y desigual. 


\section{Intergubernamentalidad}

El nivel de las coordinaciones existente entre los diferentes niveles de gobierno se comenzó a hacer evidente al momento de pactar las entrevistas. Mientras que los funcionarios nacionales entrevistados sí conocían la Iniciativa Aprende Saludable (pertenecían a la Comisión Multisectorial), los funcionarios regionales solo reconocían los programas que la componen, sea el Programa de Salud Escolar, en el caso de los de Salud, o Qali Warma, en el caso de los de Desarrollo e Inclusión Social. Los entrevistados en la primera etapa del trabajo de campo reafirmarían esta percepción inicial. Por ejemplo, uno de ellos dijo:

Creo que ya existe una coordinación a nivel nacional, pero falta todavía muchísimo en coordinación regional, y a nivel de la escuela aún más, ¿̨no? (Minedu).

Otros entrevistados fueron más allá, haciendo referencia a que había regiones en las que Aprende Saludable no se ha implementado como tal o en las que se ha implementado pero que los funcionarios regionales no han sabido enfrentar los retos que supone la coordinación intergubernamental. Refiriéndose, por ejemplo, al caso de los directores regionales, una funcionaria comentó:

[...] al final los directores regionales - a veces siempre se conversa - tienen esta dicotomía entre si estoy bajo el Gobierno regional, que es lo normativo; o si sigo una política sectorial, que es lo que en la práctica pasa más. Entonces, va a depender mucho de la orientación sectorial también y de la fuerza que le den para que lo reconozcan, ¿no? (Midis).

En las regiones se pudo comprobar algunas de estas dinámicas. Especialmente, llamó la atención la poca claridad que los funcionarios tienen respecto a la Iniciativa. Así, en una de las localidades en las que mejor funciona, una especialista no tenía clara la diferencia entre Aprende Saludable y el Plan de Salud Escolar. De aquí también se desprende que las coordinaciones se pueden llevar a cabo independientemente del conocimiento que se tenga sobre los detalles de la Iniciativa:

El Plan de Salud. No, es casi lo mismo. Si bien es cierto es el Plan de Salud Escolar, dentro de este plan está Aprende Saludable, es el marco de Aprende Saludable (DRE-Ayacucho).

Más allá de estas falencias en el flujo de información desde el centro hacia las regiones, este estudio ha podido corroborar que, en el caso de la Iniciativa Aprende Saludable, las barreras para la coordinación se desarrollan únicamente entre los sectores, no entre los niveles de gobierno.

Para que exista una disputa entre niveles de gobierno, estos tienen que tener fuertes diferencias programáticas o ejecutivas. Es decir, tienen que plantearse 
distintas políticas que se confronten entre sí o, por lo menos, deben tener distintas herramientas de gestión. En el caso peruano tal situación no existe. La ausencia de élites regionales con proyectos políticos y educativos diferentes al de Lima, y el carácter estándar de las herramientas administrativas con el que las burocracias regionales y nacionales trabajan, permite que no se genere una pugna entre las direcciones regionales y el Minedu.

En relación con esto, la ausencia de pugnas entre el nivel central y los gobiernos subnacionales alrededor de la Iniciativa Aprende Saludable también puede ser entendida por el carácter procedimental de la descentralización en el Perú, especialmente la que ha tenido lugar en el sector educación. Así, por ejemplo, Cuenca (2013) trabajó el caso de la región Arequipa, en la que este proceso implicó una reforma sectorial del Gobierno regional y el desarrollo del Proyecto Educativo Regional, así como de una propuesta de currículo para la educación básica. Estas medidas, aunque importantes, no fueron pensadas en torno a buscar un impacto directo en la niñez, sino en cumplir con determinadas pautas marcadas por el proceso. De ahí que variables de corte societal, por un lado, y otras de carácter institucional, por el otro, libran de grandes trabas a la coordinación intersectorial.

Las dinámicas conflictivas entre el nivel nacional y el subnacional también pueden desarrollarse cuando los intereses de los políticos locales se ven amenazados por las políticas nacionales. Sin embargo, a lo largo del trabajo de campo tampoco se encontraron ese tipo de disputas. Dado que el Plan de Salud Escolar y Qali Warma son desarrollados y financiados por el Estado, y que la labor de los directores en Aprende Saludable, hasta el momento, no ha requerido de una intervención decidida del Gobierno regional —o, incluso, de un financiamiento-, los Gobiernos regionales han tendido a ver la Iniciativa con indiferencia.

De esta manera, otras instancias del Gobierno regional tampoco han puesto mayores trabas a la coordinación con los ministerios. Es así que, por ejemplo, los gerentes de desarrollo social, por encima de las direcciones regionales de salud y educación, solamente han sido informados de la existencia de Aprende Saludable y, en el mejor de los casos, han convocado a las reuniones de los mecanismos de coordinación de la Iniciativa. La razón de esta actitud está relacionada con la falta de políticas educativas propias y con el carácter neutro de Aprende Saludable que se ha hecho mención líneas arriba. También lo está con otro hecho que ha sido permanentemente mencionado en todas las regiones por casi todos los funcionarios entrevistados: la falta de estabilidad de los cargos a este nivel. Sobre ello, una entrevistada cuenta: 
A: Pero cuál es el problema ahorita, el problema es que el cambio, como te digo, el cambio político; estás empezando a trabajar con uno, todos cambian y otra vez empezar, ¿no? [...] Son tres gerentes de desarrollo.

E: ¿En este año?

A: En este año (Diresa-Ucayali).

Pese a esto, los burócratas regionales, específicamente los especialistas de las direcciones regionales de salud y educación — quienes están llamados a implementar los mandatos que vienen desde la capital—, asumen, al igual que los funcionarios del nivel nacional, que una política que implique la colaboración entre los diversos sectores es importante. Así, por poner un ejemplo, una funcionaria reflexiona sobre la integralidad que implica la Iniciativa:

Y yo veo esta Iniciativa que hace algo, este, se juntan los tres sectores, ¿̨no? O sea, tenemos esta población de niños, el objetivo para cada uno de nosotros, ¿̨no?, y cómo mejoramos y cuál es el objetivo final, que los niños aprendan saludablemente. Obviamente para el desarrollo integral, viendo el desarrollo integral del nińo. No, si a mí me parece algo acertado (Diresa-Ayacucho).

En esa línea, a lo largo de las entrevistas realizadas se notó una fuerte dependencia del personal regional de cada uno de los sectores a los ministerios. Lejos de existir una cercanía con las autoridades regionales, más bien fue muy evidente que se sentían más cercanos y comprometidos con el nivel nacional y sus iniciativas. De esa manera, la percepción en todas las regiones visitadas fue de tener al frente a funcionarios nacionales a los cuales la región solamente se dedicaba a pagarles y cobijarlos en sus instalaciones. Tal situación ha ayudado en gran medida a evitar que se generaran muchas más barreras en la coordinación.

La ausencia de dinámicas intergubernamentales también está relacionada con la ausencia de la Iniciativa en el nivel local. Pese a que varios de los entrevistados consideran importante que los Gobiernos locales estén integrados a Aprende Saludable, dado que pueden facilitar las labores de difusión de información en torno a la Iniciativa, la vigilancia de un entorno saludable alrededor de las escuelas (evitando la presencia de vendedores de alimentos con alto contenido de azúcares y grasas) y algunas mejoras en la infraestructura que permitan realizar un mejor trabajo a los tres sectores en general; en pocas regiones que se visitó, este nivel de gobierno tenía un rol preponderante dentro de las labores de coordinación. Solo en algunas regiones se había empezado recién algunas coordinaciones. Respecto a ello, una funcionaria regional narra cómo se ha llevado a cabo: 
E: ¿Las municipalidades estarían dispuestas a colaborar con Aprende Saludable?

A: A ver, nosotros hemos diseñado una estrategia, hemos firmado con ellos acuerdos de gestión territorial, entonces, los hemos convocado. Tenemos 128 distritos, los hemos convocado, solamente se han interesado 62 [...] [En los informes se les solicita que] trabajen el tema de quioscos escolares. Cosas como esas, que sí las pueden llegar a cumplir, y les hemos planteado metas a los cuatro años de gestión y una estrategia. Bueno, recién es un proceso que lo estamos iniciando, pero que ahí la respuesta que hemos obtenido de ellos, incluso ellos mismos están «oye, ¿ya?» y «¿cuándo nos visitan? ¿Está bien lo que yo estoy haciendo?».

E: O sea, que podría funcionar si se formaliza un poco más.

A: Sí, sí, sí. Yo creo que sí (Diresa-Lima Provincias).

De esa manera, pese a la relación armónica que, en general, los entrevistados argumentaron que existía entre los ministerios y los Gobiernos regionales, el mayor reto entre estos dos estamentos se encuentra en torno a mejorar los canales de información y a emprender, de parte del Gobierno nacional, un mayor acompañamiento a las actividades de los especialistas en las regiones. Sobre lo primero, una entrevistada reflexionó en torno a la importancia del desarrollo de la base de datos en común para incentivar a una mayor coordinación entre Lima y las regiones:

[...] se sabía que había que imaginar un curso de acciones para que surgiera la lógica intersectorial a nivel regional, territorial también. Por eso era una apuesta interesante la base de datos interoperable, porque si todos teníamos eso, la posibilidad de territorializar la base, digamos, era fácil, ¿¿no? Entonces, de acá a acá es tu provincia, mira como tus chicos tienen los tres servicios, ¿no?, qué se yo, ¿no? Y estaban por construir (Minedu).

Sobre el acompañamiento que los ministerios deben realizar a los especialistas en las regiones, una especialista argumenta:

Claro. Yo no sé si esto será una problemática nacional ¿¿no? Pero en la región, el compromiso de Educación sí nos ha sido más complicado. Y como yo decía, son los docentes que se manejan mucho por normas, pero yo también digo, si una norma, si no va acompańada de algún otro proceso, que no sé si debe ser nacional o, por ejemplo, fortalecer el tema de monitoreo. "Oye estás cumpliendo lo que te dejé». Eso no se ha visto acá porque ayer, anteayer, estuvieron recibiendo un equipo a nivel nacional, pero no miran Aprende Saludable, ¿no? Entonces eso debería formar parte de su mirada como ente rector (Diresa-Lima Provincias).

En esa línea, un funcionario del Minedu menciona la importancia del monitoreo a la labor de los funcionarios del nivel subnacional a la luz del funcionamiento del sector público en el Perú:

Lo segundo que ayuda también, es que hay un equipo que permanentemente está pensando en eso. Lamentablemente, en el sector público, si dejas que una tarea sea 
solamente transversal, probablemente no ocurra [...] ¿Porque son malos funcionarios? No. ¿Porque son mala gente? No. Simplemente porque tienen otras prioridades. Entonces, en el sector público, para mover una prioridad tienes que hacer un equipito que lo mueva. Sino, es muy difícil que vaya a ocurrir por inercia (Minedu).

Dicho todo esto, antes que barreras para el desarrollo de Aprende Saludable, a partir de las características propias del proceso de descentralización en el Perú y de las regiones en sí mismas, así como por el poco interés que Aprende Saludable suscita en los Gobiernos regionales, la intergubernamentalidad ha planteado incentivos para la coordinación entre los actores involucrados en su desarrollo, los cuales pueden ser mejor aprovechados a través de un mayor involucramiento de los funcionarios del nivel nacional en los esfuerzos que realizan sus pares en las regiones. Así, las principales limitaciones que esta Iniciativa enfrenta se encuentran en la coordinación intersectorial.

\section{Conclusiones}

La literatura sobre coordinación intersectorial e intergubernamental ha tendido a señalar que, aunque la voluntad política es importante para la articulación de los diferentes sectores y niveles de gobierno, tiene serios límites para institucionalizarse. El presente trabajo ha coincidido plenamente con ello. Pese al impulso inicial de las ministras De Habich, Salas y Trivelli, la ausencia de un liderazgo activo por parte de los ministros que las sucedieron hizo que Aprende Saludable se estancara. Sin embargo, la falta de agencia dista de ser el único factor que conspiró contra esta Iniciativa.

Otro factor, señalado en la revisión teórica, que limita la coordinación en el Estado es la sectorialización. El acercamiento a Aprende Saludable ha permitido conocer más de cerca que el Estado peruano se mueve bajo una lógica sectorial, en la que los funcionarios desconfían de los demás niveles de gobierno; centralista, que hace que la instancia nacional subsuma a las instancias subnacionales; y procedimental, en la que las acciones de los funcionarios públicos está guiada por la presencia de normas escritas e instancias que garantizan su cumplimiento. Así, aunque los actores de los diferentes sectores y niveles reconocen como fortaleza la importancia de la coordinación —-dado que genera un mayor impacto en la ciudadanía, es más rentable económicamente-, en la práctica no siempre la han podido desarrollar.

A lo largo del estudio se pudo notar que los límites para la coordinación también están relacionados con la ausencia de recursos administrativos, reflejada en las múltiples responsabilidades y la excesiva rotación que muchos de los 
encargados de las coordinaciones deben de afrontar. Así, por ejemplo, los maestros son especialmente renuentes a participar en la Iniciativa, dado que implica aumentar más tareas a su abultada carga de trabajo. También se ve reflejada cuando hay una mayor rotación de gerentes y directores, lo que hace menos probable que la coordinación se lleve a cabo de forma permanente porque a los funcionarios les toma tiempo aprender de qué manera llevar a cabo la articulación entre los sectores involucrados.

Por otro lado, se halló que las potencialidades están relacionadas con las prácticas cotidianas que tienen algunos burócratas del nivel subnacional, quienes coordinan entre sí sin necesidad de alguna norma escrita de por medio, dado que muchas de sus labores están relacionadas (por ejemplo, los alimentos de Qali Warma se entregan en las escuelas, por lo que el personal de este programa de por sí ya conoce a los directores de escuela de su ámbito territorial). También están relacionadas con que los grupos de trabajo de los sectores sean multidisciplinarios, dado que, en los casos que coincidan profesionales de un mismo ramo, se generan incentivos para que coordinen de manera efectiva.

Respecto a las relaciones intergubernamentales, aunque la literatura sobre el tema resalta que, salvo algunos casos específicos como el colombiano, estas normalmente tienden a ser problemáticas, en el presente estudio se ha podido notar que el personal regional tiene una fuerte dependencia con sus sectores y los ministerios correspondientes. En ese sentido, el caso de Aprende Saludable aporta al debate sobre la coordinación gubernamental, llamando la atención que, al momento de acercarnos a los problemas de coordinación en países unitarios y altamente centralistas, hay que fijarnos predominantemente en los problemas de coordinación intersectoriales antes que en los de carácter intergubernamental. Esto podría resultar de gran utilidad para hacer menos complejas las miradas sobre este tema, lo cual permitiría tener respuestas más satisfactorias sobre los factores que la limitan y los que la favorecen.

Más allá del contexto institucional y de la cantidad de recursos administrativos que los burócratas tienen disponibles, ha resultado evidente que la normativa existente no es suficiente para institucionalizar esta Iniciativa. Los funcionarios en las regiones requieren de normativas claras que indiquen cuál es su rol como parte de Aprende Saludable y qué implicancias tiene para sus funciones. Aun cuando estas normas tuvieran un carácter simbólico, principalmente para el sector educación, su poder se asienta en el ordenamiento de las acciones, la definición de los objetivos y la claridad en las tareas. Por ello, para el éxito de futuras experiencias de coordinación gubernamental, sería muy importante apoyarlas en una institucionalidad que tenga en cuenta normas de 
funcionamiento explicitas, que considere la alta carga laboral de los funcionarios (sean profesores, médicos, entre otros) y que incentive tanto la coordinación con otros sectores como la estabilidad laboral.

\section{BibLIOGRAFÍA}

Acuña, C. (2005). Notas metodológicas para comprender (y mejorar) las características políticoinstitucionales de las estrategias de reducción de la pobreza en América Latina. X Congreso Internacional del Centro Latinoamericano de Administración para el Desarrollo sobre la reforma del Estado y de la administración pública. Congreso llevado a cabo en Santiago de Chile, Chile: CLAD.

Agranoff, R. (1997). Las relaciones y la gestión intergubernamentales. En R. Bañón y E. Carrillo (Comps.), La nueva administración pública (pp. 125-170). Madrid, España: Alianza.

Bardach, E. (1998). Getting Agencies to Work Together: The Practice and Theory of Managerial Craftsmanship. Washington, DC: The Brookings Institution.

Bryson, J. M., Crosby, B. C. y Stone, M. M. (2006). The Design and Implementation of CrossSector Collaborations: Propositions from the Literature. Public Administration Review, número especial, 44-55. doi: 10.1111/j.1540-6210.2006.00665.x

Carrillo, S. y Reátegui, L. (2012). Lecciones y desafios de la coordinación intergubernamental en el marco de la reforma descentralista. El caso de la implementación del proyecto de inversión pública del distrito de Julcán. Lima, Perú: CNE y PUCP.

Chirinos, L. (2012). Estado de las politicas de descentralización educativa. 2010-julio 2011. Lima, Perú: SUMA-USAID.

Cohen, J. M., y Peterson, S. B. (1997). Administrative decentralization: A new framework for improved governance, accountability, and performance. Cambridge, MA: Harvard University Press.

Correa, G. (2001). Descentralizar el Estado desde la región: Organizar la oferta gubernamental desde la demanda regionalllocal. Santiago de Chile, Chile: Grupo de Políticas de PNUD.

Cortázar, J.C. (2001). Oportunidades y limitaciones para la reforma del Estado en el Perú en los años 90. Lima, Perú: PUCP.

Cuenca, R. (2013). Reformas institucionales y acción social: La experiencia de la descentralización educativa en Arequipa. En A. M. Manrique (Ed.), Cátedra Arequipa. Primera sesión. Diez años de descentralización en el Perú: Balance y perspectivas (pp. 161-173). Arequipa, Perú: Instituto de Gobierno y Desarrollo Humano y Adrus DyL Editores.

Cunill, N. (2014). La intersectorialidad en las nuevas políticas sociales. Un acercamiento analíticoconceptual. Gestión y Política Pública, 23(1), 5-46.

Dye, T. R. (2008). Understanding Public Policy. Englewood Cliffs, NJ: Prentice Hall.

Galeano, M. (2009). Estrategias de investigación social cualitativa. El giro de la mirada. Medellín, Colombia: La Carreta editores.

Jordana, J. (2001). Relaciones intergubernamentales y descentralización en América Latina: Una perspectiva institucional. (Serie Documentos de Trabajo I- 22 UE). Washington DC, USA: BID.

Lindblom, C. (1991). El proceso de elaboración de politicas públicas. Madrid, España: MAP.

Meade, B. y A. Gershberg (2010). Análisis de la implementación de las reformas de descentralización y modernización en Colombia. (Documento de trabajo). Lima, Perú: USAID.

Migdal, J. (1988). Strong Societies and Weak States: State-Society Relations and State Capabilities in the Third World. Princeton, NJ: Princeton University Press. 
Ministerio de Educación (s/f). Iniciativa "Aprende Saludable», Balance 2013 y perspectivas 2014. (Documento interno no publicado, mimeo.) Lima, Perú: Minedu.

Molina, R. (2010). Experiencias de reforma institucional en gobiernos regionales. Estudio de casos. Lima, Perú: PRODES-USAID y Congreso de la República.

Muñoz, F. y Valdivia, N. (2012). Balance de descentralización. Documento interno de la Mesa Interinstitucional de Gestión y de Descentralización del Consejo Nacional de Educación (documento inédito). Lima, Perú: CNE.

Muńoz, F., Neyra, C. y Vargas, J. (2012). La gestión educativa municipal del distrito de La Matanza en Piura. Municipalidades apostando por la educación. Lima, Perú: CNE y PUCP.

Nickson, A. (1998). ¿¿Hacia dónde se dirige el gobierno local en América Latina? Una perspectiva comparativa. Caracas, Venezuela: CLAD.

Pezo, I. (2012). La experiencia de la gobernanza local en educación en el distrito de Cuñumbuqui, región San Martín, periodo 2011-2012. Lima, Perú: CNE y PUCP.

Repetto, F. (2005). La dimensión política de la coordinación de programas y politicas sociales: Una aproximación teórica y algunas referencias prácticas en América Latina. En F. Repetto (ed.), La gerencia social ante los nuevos retos del desarrollo social en América Latina (pp. 39-100). Guatemala: Indes/ BID.

Simons, H. (2012). El estudio de caso: teoría y práctica. Madrid, España: Morata.

Stake, R. (1999). Investigación con estudio de casos. Madrid, España: Morata.

Sulbrandt, J. (2005). Relaciones interorganizacionales y el Programa de Comunidades Especiales de Puerto Rico: Una experiencia de "gobierno conjunto». Santiago de Chile: Facultad Latinoamericana de Ciencias Sociales. 